\title{
Superior clinical results and higher satisfaction after customized compared with conventional TKA
}

\author{
Alexander Zeh, Valentina Gehler, Natalia Gutteck, Johannes Beckmann, Richard Brill, David Wohlrab
}

From the University Hospital Halle, Germany

Investigation of functional outcome and patient's satisfaction after implantation of a customized versus conventional TKA. In 31 consecutively enrolled patients with primary gonarthrosis, 33 customized TKA (custTKA) and in 31 patients, a conventional TKA (convTKA) was implanted. Perioperative and postoperative management were identical. Radiographic evaluation, ROM, KSS (Knee society score) and WOMAC (Western Ontario and McMaster Universities Osteoarthritis Index) were performed and patients satisfaction was evaluated after 3 and 12 months. Groups were comparable for age, sex, body mass index and extension/flexion.

After 92 days average flexion in the convTKA group was significantly higher (119 vs. 113 degrees; unpaired t-test). At 375 days, mean flexion in both groups was 120 degrees. There was a significant higher number of outliers of neutral mechanical axis for convTKA patients (11 vs. 3; Chi-squared test). After 92 days there was no difference for KSS (convTKA: 160, custTKA: 167) but significant better results for WOMAC (19 vs. 40) in the custTKA group (unpaired t-test, $p=0.02)$. In addition, significantly better KSS (181 vs. 156) and WOMAC (99 vs. 42) were found for the custTKA group at 375 days (unpaired t-test, $p=$ 0.002 and 0.001). Patients with the custTKA implant reported significant higher fulfillment of their expectations regarding function and knee strength. In the present study, the patients with a custTKA implant showed significantly superior short-term clinical results and fulfillment of their expectations regarding knee function.

All authors have disclosed any financial competing and any non-financial competing interests.

Johannes Beckmann receives honoraria from DePuy, Smith \& Nephew and Conformis which, however, does not conflict with study interests.
Keywords: customized implants; individual; total knee arthroplasty; outcome; off-the-shelf implants; patients expectations.

\section{INTRODUCTION}

Despite total knee arthroplasty providing significant improvements in function and pain for the majority of patients with knee arthritis, a significant number of patients are not satisfied with their results $(1,2)$. Dissatisfaction rates are reported to be as high as $19 \%$ with patients citing either a lack of pain relief or functional improvement as being the reason for dissatisfaction (3). Continuing advances in implant design have attempted to improve function and kinematics. Though newer generations of CR or PS designs, fixed or mobile bearing, design features

\footnotetext{
Alexander Zeh ${ }^{1}$,

Valentina Gehler ${ }^{1}$,

Natalia Gutteck ${ }^{1}$,

Johannes Beckmann²,

- Richard Brill' ${ }^{1}$

- David Wohlrab

'Department of Orthopedic Surgery and Traumatology, University Hospital Halle (Saale), Halle (Saale), Germany. ${ }^{2}$ Sportklinik Stuttgart, Germany.

Correspondence : Alexander ZEH, Department of Orthopedic and Trauma Surgery, Ernst-Grube-Strasse 40, 06120 Halle, Germany. Phone: +49 (0)345 557 4805, Fax: +49 (0)345 5571329.

Email : alexander.zeh@uk-halle.de

- 2021, Acta Orthopædica Belgica.
} 
have been introduced to improve flexion, and gender specific prostheses have been introduced, to improve implant fit, the ideal design for TKA remains undefined (4).

Conventional TKA geometries are based on averaging dimensions obtained from CT data. However, these by nature, due to the limited sizes of the implants, represent a compromise between the offered size ranges and the widely varying anatomic geometries and sizes. These findings suggest that hypothetically a greater degree of customization could result in surgeons performing fewer soft tissue releases and medial resections than are being done to fit a fixed-geometry. However, these imaging studies cannot support one approach to TKA over another; comparative will be required to proof advantages of customized TKA (5). It is well established that implant overhang (6) and anteroposterior oversizing (7) negatively impact the clinical outcome. Individual differences of J-Curve and distal and posterior condylar offset are assumed to have influence on the postoperative result if they are not individually reconstructed (8). Thus, customization of TKA has been introduced to address these anatomical variations, with the aim of recreating physiological kinematics after implantation $(9,8,10)$. However, it is still unclear to which extend individual variation in anatomy and kinematics needs to be restored to improve functional results (11).

Also, despite encouraging clinical and biomechanical results of patient specific implants $(10,12,13)$ clinical studies $(10,14,15)$ to proof superiority of this technique are rare or even report controversial results (16). Furthermore, postoperative satisfaction is multifactorial and many factors have been isolated which may have influence, e.g. age, gender, comorbidities, expectations (17).

Therefore, study-in-hand wanted to investigate prospectively and consecutively whether clinical, radiological results and patient-reported outcome for patients implanted with a custTKA differ compared to a conventional TKA system.

\section{MATERAIL AND METHODS}

From 01/2016 to 03/2017 62 patients with the diagnosis of primary gonarthrosis received a cemented bicondylar posterior stabilized total knee arthroplasty (TKA) and were prospectively and consecutively enrolled. In 31 of these patients, 33 customized TKA (iTotal PS, ConforMIS Europe GmbH, Fürth, Germany) (custTKA) were implanted while the other 31 patients received 31 a conevtional TKA (Genesis II PS, Smith\&Nephew GmbH, Hamburg, Germany) (convTKA).

All patients included in this analysis suffered from arthritic pain, functional deficits and impaired quality of life, with previously failed conservative therapy. Each group was operated on by a single experienced senior surgeon. Patients were included as consecutive cases as one surgeon used either one technique throughout the enrollment process for the study.

A medial parapatellar approach without tourniquet was performed in all cases. Both systems were implanted according to manufacturer's manual using the single-use PSI cutting blocks and customized instrumentation based on CT scan data in the custTKA group.

In the convTKA group intramedullary referencing was performed and standard metal cutting blocks were utilized to prepare the joint. Both prostheses were planned and implanted to achieve a neutral leg alignment 0 degrees. For implantation of the convTKA a tibial slope of 3 degrees and neutral alignment of the femoral implant with regard to extension/flexion was targeted. In the custTKA a neutral slope and typically 5 degrees of flexion of femoral prosthesis to avoid notching the anterior cortex were aimed.

All knees should have a symmetric medial and lateral joint space and balanced ligament tension in both extension and flexion. Therefore, stepwise medial and lateral ligament balancing was performed as necessary. An intraarticular drain was used in all cases for 24 hours. All patients obtained a perioperative single shot antibiosis preoperatively and subcutaneous low-dose heparin for 14 days postoperatively.

An intraoperative LIA with $150 \mathrm{ml} 0.2 \%$ Ropivacain and $0.5 \mathrm{ml}$ Adrenalin 1:1000 with infiltration of the ligaments, dorsal capsule, fat pad, capsule incision and subcutaneous tissue was performed at the end of surgery. In addition, all patients were 
Table I. - Questions about patients satisfaction

\begin{tabular}{|l|c|l|}
\hline Would you undergo this surgery again? & $0-10$ & I absolutely agree/disagree \\
\hline Were your expectations regarding pain reduction fulfilled? & $0-10$ & I absolutely agree/disagree \\
\hline Were your expectations regarding step stairs fulfilled? & $0-10$ & I absolutely agree/disagree \\
\hline Were your expectations regarding flexion fulfilled? & $0-10$ & I absolutely agree/disagree \\
\hline Were your expectations regarding knee strength fulfilled? & $0-10$ & I absolutely agree/disagree \\
\hline
\end{tabular}

Each of these questions could be marked with a cross in a box counted from 0 to 10 according to "I absolutely agree.... I absolutely disagree".

administered $3 \times 600 \mathrm{mg}$ Ibuprofen during the

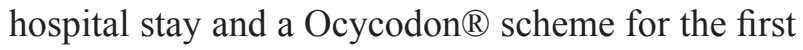
three postoperative days. The scheme consisted of $10 \mathrm{mg}$ Ocycodon p.o. every 12 hours and up to $5 \mathrm{mg}$ on demand every 2-4 hours. From the fourth postoperative day Oxycodon scheme was finished and pain management was supplemented by Novalgin ${ }^{\circledR} 3 \times 20$ drops on demand.

Surgical time (defined as time from first skin incision to wound closure), blood loss (Hb balance method (18-20), along with pre-operative Knee Society Score (KSS) (21) were recorded.

The KSS contains questions in 2 sections: knee joint (pain, range of motion, stability) and function (walking distance, ability to climb stairs). Deductions are taken for assistive devices and flexion contractures, misalignment, or extension lag. The maximum summarized score of both section is 100, indicating the best result (21).

Following an established procedure at our department, all patients were discharged at 5 days postoperative. All patients followed the same physiotherapy concept, which included full weight bearing from the first postoperative day, crutches, continuous passive motion (CPM), special walking training and a three week inpatient rehabilitation after discharge from hospital, which is standard of care in the German healthcare system.

Lateral-view and a. p. radiographs were obtained before discharge and 3 months postoperatively using a standardized X-ray protocol. Additionally, digital long-leg standing anteroposterior radiographs were obtained 3 or 12 months postoperatively. KSS and WOMAC (Western Ontario and McMaster Universities Osteoarthritis Index) (22) were obtained
3 and 12 months postoperatively. Complications were documented.

The WOMAC was obtained as a questionnaire consisting of five questions for pain, two questions for stiffness and 17 questions for physical function each could be marked with a cross from 0 to 10 . Therefore, the best result would be 0 and the worst result a score of 240 .

In addition, individual questions were asked to report patient's satisfaction after surgery. Each of these questions could be marked with a cross in a box from 0 to 10 (Table I). Similar to the WOMAC Score the best result of each question would be 0 and the worst result 10 .

\section{Statistical analysis}

Continuous data are presented as mean and standard deviation (sd). Inter-group comparisons were performed using an unpaired t-test. In case of violations of the normality assumption, inter group comparisons were performed using the MannWhitney-U-Test. Chi-squared test was performed to determine whether there is a significant difference between observed frequencies of certain categories in both groups.

A $p<0.05$ was used to determine significance between measured data between the two groups.

For random number calculation of the unpaired t-test a clinically relevant difference of postoperative flexion of 8 degrees was determined. A standard deviation of 10 degrees was assumed. A minimum number of cases of 25 in each group was calculated ( $a p h a=0.5$, beta $=0.8$, double-sided significance). Statistical analysis was performed with SPSS 25 
Table II. — Preoperative data

\begin{tabular}{|c|c|c|}
\hline Parameter & $\begin{array}{l}\text { custTKA } \\
\text { group }\end{array}$ & $\begin{array}{l}\text { convTKA } \\
\text { PS group }\end{array}$ \\
\hline $\mathbf{N}$ (implants) & 33 & 31 \\
\hline Male/female & $\mathbf{1 4 / 1 7} \mathrm{p}^{\mathrm{p}^{*}=0.9}$ & $13 / 18$ \\
\hline Age & $\mathbf{6 9 . 3} 3^{\mathrm{p}=0.9} \quad(9)$ & $69.1 \quad(10)$ \\
\hline BMI & 31.5 $5^{\mathrm{p}=0.1} \quad(5)$ & $29.6 \quad(5)$ \\
\hline Preop. extension & $\mathbf{- 0 . 8 ^ { p = 0 . 3 }} \quad(2.2)$ & $-\mathbf{- 1 . 5} \quad(3.7)$ \\
\hline Preop. flexion & $109^{\mathrm{p}=0.08} \quad(16)$ & 105 \\
\hline Preop. pain** & $\mathbf{1 0 . 6}^{\mathrm{p}=0.4} \quad(12.1)$ & $(9.3)$ \\
\hline KSS & $\mathbf{1 0 0}^{\mathrm{p}=0.02} \quad(35)$ & (24) \\
\hline KSS Knee Score (A) & $\mathbf{4 6}^{\mathrm{p}=0.3} \quad(15)$ & $(15)$ \\
\hline KSS Function Score (B) & $\mathbf{5 4} \mathbf{4}^{\mathrm{p}=0.001} \quad(20)$ & $46 \quad(14)$ \\
\hline
\end{tabular}

mean values, (data in brackets) $=$ standard deviation, $\mathrm{p}^{*}=$ result of Chi-Square test, $\mathrm{p}=$ result of unpaired t-test, $\mathrm{BMI}=$ Body mass index, KSS= Knee Society Score, Preop. = preoperative, **preoperative pain extracted from KSS part A (points).

(IBM SPSS Statistics, v. 20, Illinois, Chicago, USA).

\section{RESULTS}

Both cohorts were found to be similar in major preoperative variables that were observed (Table II). The only statistically significant difference was in the preoperatively measured KSS, with a higher total Score for the convTKA group. This result was attributable to significantly higher values for the Function Score of the KSS while there was no difference in the objective Knee Score between the two groups. Table II shows preoperative baseline data. Unpaired t-test was significant for the KSS only.

The average operating time was significantly longer in the custTKA group (56 minutes +/- 11 minutes versus 49 minutes $+/-8$ minutes in the convTKA group; $\mathrm{p}=0.008$ for unpaired t-test).

The femorotibial angle measured between the anatomical axis of the femur and tibia was $5^{\circ}(\mathrm{sd}$ : $\left.2^{\circ}\right)$ of varus in the custTKA group and $6^{\circ}\left(\mathrm{sd}: 4^{\circ}\right)$ of valgus in the convTKA group. This result was not statistically different ( $\mathrm{p}=0.1$ for unpaired t-test). The measurement of the mechanical axis in long leg
Table III. - Outliers from $+/-3$ degrees of mechanical femorotibial axis in long standing X-rays

\begin{tabular}{|l|c|c|}
\hline Outliers & $\begin{array}{c}\text { custTKA } \\
33 \text { implants }\end{array}$ & $\begin{array}{c}\text { convTKA } \\
31 \text { implants }\end{array}$ \\
\hline$-\mathbf{4}^{\circ}$ & & 2 \\
\hline$-\mathbf{5}^{\circ}$ & & 1 \\
\hline$-\mathbf{6}^{\circ}$ & & 2 \\
\hline $\mathbf{- 9 ^ { \circ }}$ & 2 & 1 \\
\hline$+\mathbf{4}^{\circ}$ & & 4 \\
\hline$+\mathbf{5}^{\circ}$ & 1 & 1 \\
\hline$+7^{\circ}$ & & \\
\hline+
\end{tabular}

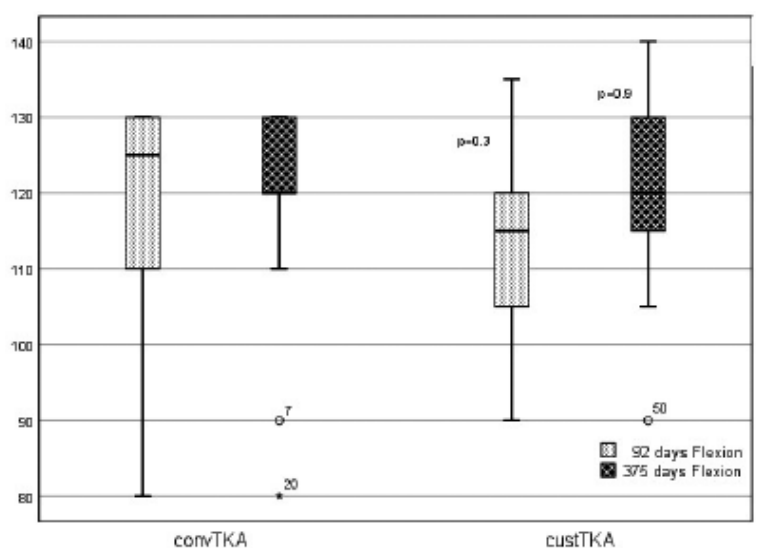

Figure 1. - Box plot: Knee flexion after 92 and 375 days postoperatively. $\mathrm{p}=$ result of unpaired t-test.

standing $\mathrm{x}$-rays after 5.8 months postoperatively on average resulted in $1.5^{\circ}$ of varus $\left(2^{\circ}\right.$ valgus to $7^{\circ}$ varus) for the custTKA group and $1^{\circ}$ of valgus $\left(9^{\circ}\right.$ valgus to $5^{\circ}$ varus) for the convTKA group which is a statistically significant difference $(p=0.001$ for unpaired t-test).

In the convTKA group, there were a total number of 11 outliers from $+/-3$ degrees whereas in the cust TKA group three outliers were observed (see Table III).

The Chi-Square-test showed no statistical significance $(\mathrm{p}=0.5)$.

Following the Hb-based $(19,18)$ determination we found an average total blood loss of $1447 \mathrm{ml}$ for the custTKA group and $1405 \mathrm{ml}$ for the convTKA group (unpaired t-test, $\mathrm{p}=0.5$ ). Hb-based measurement of 
Table IV. — Frequencies of Extension lack after 92 days on average

\begin{tabular}{|l|c|c|}
\hline $\begin{array}{l}\text { Degrees of } \\
\text { Extension lack }\end{array}$ & $\begin{array}{c}\text { custTKA group } \\
33 \text { implants }\end{array}$ & $\begin{array}{c}\text { convTKA group } \\
31 \text { implants }\end{array}$ \\
\hline $\mathbf{5}^{\circ}$ & 3 & 2 \\
\hline $\mathbf{1 0}^{\circ}$ & 1 & 4 \\
\hline $\mathbf{1 5}^{\circ}$ & 0 & 1 \\
\hline
\end{tabular}

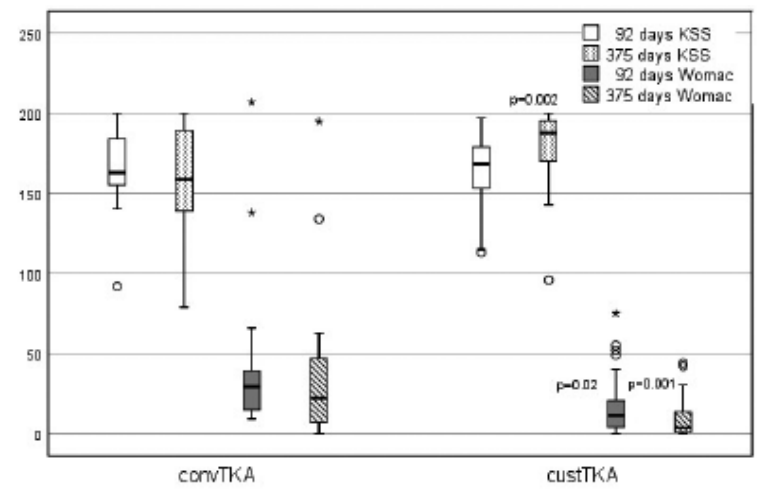

Figure 2. - KSS and WOMOC after 92 and 375 days postoperatively. $\mathrm{p}=$ result of unpaired t-test.

blood loss is regularly associated with significantly higher values than measurement of intraoperative blood loss (23).

Figure 1 shows postoperative flexion after 92 days ( $\mathrm{sd}=30$ days) and 375 days ( $\mathrm{sd}=35$ days) on average. In the custTKA group average flexion was $113^{\circ}\left(\mathrm{sd}=12^{\circ}\right)$ at 92 days while $119^{\circ}\left(\mathrm{sd}=12^{\circ}\right)$ were measured for the convTKA group. This difference was statistically significant (unpaired t-test $\mathrm{p}=0.03$ ). At 375 days on average this statistically significant difference was no longer present (custTKA group= $119,8^{\circ}\left(\mathrm{sd}=12^{\circ}\right)$; convTKA group $=120,3^{\circ}(\mathrm{sd}=$ $\left.12^{\circ}\right)$ ) (Figure 1).

After 92 days, average knee extension was $-2.2^{\circ}$ $\left(\mathrm{sd}=4.8^{\circ}, \min =0^{\circ}, \max =15^{\circ}\right)$ in the convTKA group of patients, and $0.3^{\circ}\left(\mathrm{sd}=1.25^{\circ}, \mathrm{min}=0^{\circ}\right.$, $\max =10^{\circ}$ ) for the custTKA group which was not of statistically significant (unpaired t-test, $\mathrm{p}=0.38$ ). Frequencies of extension contracture $\geq 5$ degrees are listed in table IV. The Chi-square test shows no significance $(\mathrm{p}=0.34)$.

At 92 days postoperatively no statistically significant difference was observed for KSS (convTKA: 160, $\mathrm{sd}=22$; custTKA: $167, \mathrm{sd}=12, \mathrm{p}=0.42)$.

For WOMAC (convTKA: 40, sd=46; custTKA: $19, \mathrm{sd}=19)$ the result in the custTKA group was significantly better (unpaired t-test, $\mathrm{p}=0.02$ ). In addition, significantly higher values for KSS (convTKA: $156+/-33$; custTKA: $181, \mathrm{sd}=24$ ) and better WOMAC (convTKA: 42, $\mathrm{sd}=47$; custTKA: $9, \mathrm{sd}=19$ ) were found in the custTKA group at 375 days postoperatively (Figure 2) (unpaired t-test, $p=0.002$ and 0.001 ). The statistically significant difference was present in both KSS Knee Score (convTKA: 91, $\mathrm{sd}=21$; custTKA: $95, \mathrm{sd}=4$ ) (unpaired t-test, $p=0.001$ ) as well as in the KSS Function Score (convTKA: 76, $\mathrm{sd}=48$; custTKA: 91, $\mathrm{sd}=23$ ) (unpaired t-test, $\mathrm{p}=0.006$ ).

Table V. - Questions about patients satisfaction

\begin{tabular}{|c|c|c|c|c|c|}
\hline \multirow[t]{2}{*}{ Patient satisfaction } & \multicolumn{2}{|c|}{$\begin{array}{c}\text { after } \\
92 \text { days }\end{array}$} & \multicolumn{2}{|c|}{$\begin{array}{c}\text { after } \\
375 \text { days }\end{array}$} & \multirow{2}{*}{$\begin{array}{c}\mathbf{p} \\
92 \text { days } \\
375 \text { days }\end{array}$} \\
\hline & convTKA & custTKA & convTKA & custTKA & \\
\hline Would you undergo this surgery again? & 1.5 & 0.3 & 2.0 & 0.01 & $\begin{array}{c}0.07 \\
0.001\end{array}$ \\
\hline $\begin{array}{l}\text { Were your expectations regarding pain reduction } \\
\text { fulfilled? }\end{array}$ & 1.5 & 0.4 & 2.1 & 0.4 & $\begin{array}{l}0.04 \\
0.02\end{array}$ \\
\hline Were your expectations regarding step stairs fulfilled? & 2.1 & 0.8 & 2.7 & 0.4 & $\begin{array}{c}0.04 \\
0.003\end{array}$ \\
\hline Were your expectations regarding flexion fulfilled? & 1.6 & 0.7 & 1.6 & 0.5 & $\begin{array}{c}0.08 \\
0.008\end{array}$ \\
\hline $\begin{array}{l}\text { Were your expectations regarding knee strength } \\
\text { fulfilled? }\end{array}$ & 1.7 & 0.5 & 1.6 & 0.2 & $\begin{array}{c}0.02 \\
0.008\end{array}$ \\
\hline
\end{tabular}

Each of these questions could be marked with a cross in a box counted from from 0 to 10 according to "I absolutely agree.... I absolutely disagree". 
At the follow-up after 92 days in the convTKA group 31 and in the custTKA group 32 cases could be recorded for clinical and radiographic examination. Of these in the convTKA group, 26 patients filled the KSS/ WOMAC score, whereas in the custTKA group we were able to obtain the scores in 30 cases. Unfortunately, not all patients were compliant to fill scores during the clinical visit. After 375 days on average 30 cases of the convTKA and 31 cases of the custTKA group returned for clinical and radiographic follow-up. The KSS/WOMAC was available in 29 cases of the convTKA and in 30 cases in the custTKA group after 375 days on average.

Results of individual questions to report patient's satisfaction are reported in table V. All but two questions after 92 days on average and all question after 365 days on average were answered towards higher satisfaction by custTKA patients.

Of the custTKA patients, one was excluded from the study after two revision surgeries (release and change of liner) with the diagnosis of arthrofibrosis. In another patient, a significant reduction of kidney function occurred and was treated successfully.

In the convTKA group, an embolism of the A. cerebri media occurred postoperatively with full recover and a prolonged wound healing without required revision was observed.

\section{DISCUSSION}

There are several publications investigating potential advantages an different aspects of patientsspecific customized implants to answer the question if this new and innovative technique could lead to higher rates of satisfaction and better clinical outcome after TKA. Comparative studies are still rare $(24,16,10,25-29)$.

The study-in-hand investigates prospectively if a patient-specific TKA provides better clinical results and superior alignment compared with a conventional TKA.

The main finding of the present study is that patients with custTKA showed clinical superiority in several aspects comparing with the convTKA group. In paticular, a significant higher satisfaction was observed for the custTKA regarding fulfilled expectations about postoperative pain reduction and function.
This result is in accordance with the findings of other studies on potential advantages of patientspecific implants $(15,10,30)$. However, two of these studies present clinical results for bicompartimental patient-specific TKA (15) and for unicompartimental TKA (10) in non-comparative studies. The present findings corroborate those of the study by Reimann et al. 2019, in which patients with a patient specific TKA exhibited significantly higher satisfaction rates, overall KSS and KSS Functional scores. In this study, patient-specific implants were compared to a conventional implant (other system than used in the present study) (30).

All patients of the current study suffered from failed conservative therapy for primary Gonarthrosis. Preoperative data show no statistical differences for parameters such as sex, age, BMI and preoperative flexion. This finding is important as sex, age and BMI are regarded as factors, which may influence the clinical result and satisfaction after TKA (3133). Additionally, preoperative flexion is one of the main factors which influences final post-operative flexion $(34,35)$ (Table II). Therefore, it is important to observe that there were no differences regarding preoperative flexion between study groups and values were within expected preoperative ranges (36-39).

However, the preoperative KSS in the custTKA group was significantly about $20 \%$ higher than for the convTKA patients. Analysis showed that this difference between both groups was primarily driven by the significantly higher function score in the custTKA group (Part B) which represents the patient's input (21).

Theoretically, custTKA show advantages in biomechanics compared with convTKA as kine-matics closed to healthy knee joint and better contact stresses are achieved in computational analysis (26) and in particular a more physiological function of the Post-Cam mechanism $(40,13)$. Furthermore a better stability in flexion with the absence of lift-off and more physiological axial rotation during flexion was proven in in vivo kinematics using fluoroscopy (12). Of course, these findings cannot be simply transferred to an assumption of better clinical outcome and higher patient's satisfaction. 
However, in the present study no statistical significant differences were observed for KSS but of about $50 \%$ for WOMAC after 92 days. In addition, significantly better scores for KSS (12\%) and WOMAC (45\%) were found after 375 days on average in the custTKA group when compared to the convTKA group. The difference of the score points of the WOMAC between both groups are considered to be relevant following the study results about the minimal clinically important difference for the WOMAC (17 score points) after TKA (41). This point of clinical relevance was exceeded at both time points of follow-up and was found to be nearly twice as high after 375 days on average (41).

Though the Knee and Function Scores of the KSS were significantly higher in the custTKA group, it was the KSS Function Score which showed a major difference between the convTKA and custTKA patients (Figure 2).

Due to a higher function score the custTKA patients started with a roughly $20 \%$ higher KSS preoperatively. Alattas et al. (2017) found that except greater anxiety, pre-operative pain and function were the most significant factors to predict a poorer outcome of a TKA (42). Neither preoperative flexion nor the objective part A of KSS, summarizing measured preoperative knee function and pain, showed a significant difference between both groups. In particular preoperative pain, which was extracted from point system of KSS score for pain showed no significant difference (Table II). Therefore, it is concluded that there is no evidence for a relevant or proven significant difference between both groups regarding preoperative HRQoL (Health-Related Quality of Life).

In contrast the WOMAC did not improve for the convTKA group between 92 and 375 days, whereas we found a significant and clinically relevant improvement (41) for the custTKA group over the same time period. In particular the high KSS Functional Score which is higher than some previously reported results (43) in combination with the better WOMAC scores, could be indicative of a trend towards a significantly higher satisfaction in the custTKA group.

In addition, a significant higher satisfaction was observed for the custTKA documented by positive answer of main questions regarding fulfilled expectations about postoperative pain reduction and function (Table V).

This result confirms excellent results of patients satisfaction of other observers for patient-specific implants $(15,10)$. However, these studies present clinical results for bicompartimental patient-specific TKA (15) and for unicompartimental TKA (10) in noncomparative studies. Furthermore, the answering of the individual questions on satisfaction in this study resulted in a high postoperative satisfaction in both groups with small and therefore questionable clinical relevant differences.

ROM after TKA and in particular sufficient flexion is one of the most important indexes in determining clinical outcomes. Many daily activates require a knee with a flexion of greater than $90^{\circ}$ and some special activities, such as squatting for certain religious needs (need $>120^{\circ}$ ) and kneeling during prayer (need $>135^{\circ}$ ), require much greater knee joint flexion. However, patients usually do not achieve satisfactorily high degrees of flexion after TKA, although the clinical outcomes achieved by the majority of modern TKA designs are satisfactory for walking ability. Moreover, patients who had good preoperative ROM often lose deep flexion (defined as flexion $>120^{\circ}$ ) after TKA (44).

In the present study, the analysis of postoperative flexion results showed, that a statistically higher flexion of $119^{\circ}$ was achieved faster in the convTKA group compared with $113^{\circ}$ in the custTKA group at 92 days. However, this statistical difference was not present after approximately one year while both groups flexed approximately 120 degrees. This faster achievement of 120 degrees of flexion after approximately 12 weeks is interpreted as significant as usually a flexion of 110 after degrees is expected after 8 weeks (45). It is concluded that both groups achieved this flexion potential and obviously the of about five degree lower degree of flexion in the custTKA group did not negatively influence the score based measured postoperative clinical result and the observed flexion (Figure 2, Table V). In addition, the custTKA group reported a significant higher postoperative satisfaction regarding knee strength, pain reduction and stepping stairs (Table V) after 92 as well as after 375 days. 
We observed no difference regarding blood loss between the convTKA and custTKA groups. This is in contrast to the findings of others, where the rate of transfusion was found to be significantly higher in an convTKA group versus a custTKA group (14, 46).

Theoretically, a reduced blood loss with the patient specific implant is assumed mainly because of the absence of preparation of the femoral and tibial canal and a more precise fit which leads to reduced under coverage of exposed bone $(46,47,8)$. Culler et al. (2017) reported a lower transfusion rate in a customized TKA group as part of an retrospective analysis. However, the transfusion regime in that study was not communicated and it is well documented that transfusion rates differ widely as transfusion requirements vary greatly by surgeon and hospital (14).

In this study, no clinically relevant statistical difference was found regarding the axial alignment in the frontal plane. The femorotibial angle measured 5.8 months postoperatively in long leg standing $\mathrm{x}$-rays showed no statistically significant differences. However, measurement of the mechanical axis resulted in $1.5^{\circ}$ of varus for custTKA and $1^{\circ}$ of valgus for convTKA which is significantly different. Despite the statistical significance, this result is regarded to be clinically not relevant as both groups were well within $+/-3^{\circ}$ of neutral on average. Nevertheless, there was a higher but not significant total number of 11 outliers from $+/-3^{\circ}$ in the convTKA group compared to just 3 in the custTKA group (Table III).

The debate about the tolerable deviation of the mechanical axis from 0 degrees is ongoing, with growing evidence that an deviation greater 3 degrees is perhaps not as critical to the clinical outcome and long term implant survival as originally postulated (48). Additionally, the literature on residual varus would suggest that this type of outlier may be more acceptable (49). Despite these results and the historic postulation of an alignment of TKA within of $+/-3$ degrees (50) for most surgeons, neutral mechanical axis typically remains a central goal of TKA surgery (49). Authors do not believe that the outliers under 4-5 degrees of varus are critical (48). Outliers of 7 degrees of varus (observed once in each group) and a valgus malalignment over 4-5 degrees (4 in the convTKA group) are seen as more critical. Because of the small study population, the significant difference of outliers in valgus are not interpreted of clinical relevance when discussing the advantages of both TKA systems. These differences could be a consequence of individual surgical technique as well as the use of PSI in combination with the custTKA (51).

There are limitations to this study. Though a sample size calculation was performed to power the study to detect differences in postoperative flexion, we report on a relatively small number of patients. The results can be interpreted as a direct comparison between the convTKA and custTKA only and conclusion for other implants is speculative. The importance of further studies is underlined by the fact that we are faced with a huge variety of implants and surgical techniques in TKA which makes it very difficult to define major outcome parameters that could be compared across other studies. Trials usually comparatively analyze results of two or three implants with results that cannot be generally transferred unless meta-analyzes are available.

One could speculate if the absence of randomization would be a limitation. In authors opinion, randomization in implant studies reveals several problems. Blinding in an implant study would be very challenging given that both implants are readily available commercially and patents are often unwilling to undergo randomization on products that are so readily available. Conversely, a non-blinded randomized study may elicit a positive or negative response to a randomization result, whereby the provided implant type could significantly influence the patient expectation counteracting the aim of randomization. Given the consecutive nature of our enrollment into this study, and the fact that the preoperative metrics are either non-significantly different, or favored the convTKA cohort that potential impact is minimized. In addition, flexion and KSS Knee Score are objective parameters and outcome measures, which after a long period of time are unlikely to be influenced by patient's attitude towards an implant type or surgical technique.

Another limitation could be seen in the circumstance that not one single surgeon performed all 
TKAs. However, this could be also considered as a strength as surgery in each group was performed by the same experienced surgeon who was well acquainted with the implant system. Furthermore, the hospital with peri- as well as postoperative setup was identical.

\section{CONCLUSION}

In the present study the custTKA showed statistical significantly superior early clinical results in terms of patient's satisfaction and function (KSS and WOMAC) compared to convTKA.

\author{
Abbreviations \\ custTKA customized TKA \\ convTKA conventional TKA \\ KSS Knee society score \\ WOMAC Western Ontario and McMaster Universities Osteo- \\ arthritis Index \\ CPM continuous passive motion \\ LIA local infiltration analgesia \\ sd standard deviation
}

\section{REFERENCES}

1. Noiseux NO, Callaghan JJ, Clark CR, Zimmerman MB, Sluka KA, Rakel BA. Preoperative predictors of pain following total knee arthroplasty. J Arthroplasty 2014; 29: 1383-7.

2. Parvizi J, Nunley RM, Berend KR, et al. High level of residual symptoms in young patients after total knee arthroplasty. Clin Orthop Relat Res 2014; 472: 133-7.

3. Bourne RB, Chesworth BM, Davis AM, Mahomed NN, Charron KDJ. Patient satisfaction after total knee arthroplasty: who is satisfied and who is not? Clin Orthop Relat Res 2010; 468: 57-63.

4. Dall'Oca C, Ricci M, Vecchini E, et al. Evolution of TKA design. Acta bio-medica Atenei Parmensis 2017; 88: 17-31.

5. Meier M, Zingde S, Steinert A, Kurtz W, Koeck F, Beckmann J. What Is the Possible Impact of High Variability of Distal Femoral Geometry on TKA? A CT Data Analysis of 24,042 Knees. Clin Orthop Relat Res 2019; 477: 561-70.

6. Bonnin MP, Schmidt A, Basiglini L, Bossard N, Dantony E. Mediolateral oversizing influences pain, function, and flexion after TKA. Knee Surg Sports Traumatol Arthrosc 2013; 21: 2314-24.

7. Vaidya SV, Ranawat CS, Aroojis A, Laud NS. Anthropometric measurements to design total knee prostheses for the Indian population. $J$ Arthroplasty 2000; 15: 79-85.
8. Schwechter EM, Fitz W. Design rationale for customized TKA: a new idea or revisiting the past? Current reviews in musculoskeletal medicine 2012; 5: 303-8.

9. Maniar RN, Singhi T. Patient specific implants: scope for the future. Current reviews in musculoskeletal medicine 2014; 7: 125-30.

10. Steinert AF, Beckmann J, Holzapfel BM, Rudert M, Arnholdt J. Bikompartimenteller individualisierter Kniegelenksersatz Einsatz patientenspezifischer Implantate und Instrumente (iDuo ${ }^{\mathrm{TM}}$ ). Oper Orthop Traumatol 2017; 29: 51-8.

11. Rivière C, Iranpour F, Auvinet E. et al. Alignment options for total knee arthroplasty: A systematic review. Orthopaedics \& traumatology, surgery \& research OTSR 2017; 103: 1047-56.

12. Zeller IM, Sharma A, Kurtz WB, Anderle MR, Komistek RD. Customized versus Patient-Sized CruciateRetaining Total Knee Arthroplasty: An In Vivo Kinematics Study Using Mobile Fluoroscopy. J Arthroplasty 2017; 32: 1344-50.

13. Koh Y-G, Son J, Kwon O-R, Kwon SK, Kang K-T. Patient-specific design for articular surface conformity to preserve normal knee mechanics in posterior stabilized total knee arthroplasty. Biomed Mater Eng 2018; 29: 40114.

14. Culler SD, Martin GM, Swearingen A. Comparison of adverse events rates and hospital cost between customized individually made implants and standard off-the-shelf implants for total knee arthroplasty. Arthroplasty today 2017; 3: 257-63.

15. Ogura T, Le K, Merkely G, Bryant T, Minas T. A high level of satisfaction after bicompartmental individualized knee arthroplasty with patient-specific implants and instruments. Knee Surg Sports Traumatol Arthrosc 2018.

16. White PB, Ranawat AS. Patient-Specific Total Knees Demonstrate a Higher Manipulation Rate Compared to "Off-the-Shelf Implants". J Arthroplasty 2016; 31: 107-11.

17. Williams DP, O'Brien S, Doran E, et al. Early postoperative predictors of satisfaction following total knee arthroplasty. The Knee 2013; 20: 442-6.

18. Johansson T, Engquist M, Pettersson L-G, Lisander B. Blood loss after total hip replacement: a prospective randomized study between wound compression and drainage. J Arthroplasty 2005; 20: 967-71.

19. Johansson T, Lisander B, Ivarsson I. Mild hypothermia does not increase blood loss during total hip arthroplasty. Acta Anaesthesiol Scand 1999; 43: 1005-10.

20. Nadler SB, Hidalgo JH, Bloch T. Prediction of blood volume in normal human adults. Surgery 1962; 51: 224-32.

21. Insall JN, Dorr LD, Scott RD, Scott WN. Rationale of the Knee Society clinical rating system. Clin Orthop Relat Res 1989: 13-4.

22. Stucki G, Meier D, Stucki S, et al. Evaluation einer deutschen Version des WOMAC (Western Ontario und McMaster Universities) Arthroseindex. Z Rheumatol 1996; 55: 40-9. 
23. Gao F-Q, Li Z-J, Zhang K, Sun W, Zhang H. Four Methods for Calculating Blood-loss after Total Knee Arthroplasty. Chin Med J 2015; 128: 2856-60.

24. Noble PC, Conditt MA, Cook KF, Mathis KB. The John Insall Award: Patient expectations affect satisfaction with total knee arthroplasty. Clin Orthop Relat Res 2006; 452: 35-43.

25. Schroeder L, Martin G. In Vivo Tibial Fit and Rotational Analysis of a Customized, Patient-Specific TKA versus Off-the-Shelf TKA. J Knee Surg 2018.

26. Koh Y-G, Lee J-A, Chung PK, Kang K-T. Computational analysis of customized cruciate retaining total knee arthroplasty restoration of native knee joint biomechanics. Artificial organs 2018.

27. Kang K-T, Son J, Kwon O-R, Koh Y-G. Malpositioning of Prosthesis: Patient-specific Total Knee Arthroplasty Versus Standard Off-the-Shelf Total Knee Arthroplasty. Journal of the American Academy of Orthopaedic Surgeons. Global research \& reviews 2017; 1: e020.

28. Haritinian EG, Pimpalnerkar AL. Computer Assisted Total Knee Arthroplasty: Does it Make a Difference? Maedica 2013; 8: 176-81.

29. Theodore W, Twiggs $\mathbf{J}$, Kolos $\mathbf{E}$, et al. Variability in static alignment and kinematics for kinematically aligned TKA. The Knee 2017; 24: 733-44.

30. Reimann P, Brucker M, Arbab D, Lüring C. Patient satisfaction - A comparison between patient-specific implants and conventional total knee arthroplasty. J Orthop 2019; 16: 273-7.

31. Sveikata T, Porvaneckas N, Kanopa P, et al. Age, Sex, Body Mass Index, Education, and Social Support Influence Functional Results After Total Knee Arthroplasty. Geriatr Orthop Surg Rehabil 2017; 8: 71-7.

32. Aalund PK, Glassou EN, Hansen TB. The impact of age and preoperative health-related quality of life on patientreported improvements after total hip arthroplasty. Clin Interv Aging 2017; 12: 1951-6.

33. Giesinger JM, Loth FL, MacDonald DJ, et al. Patientreported outcome metrics following total knee arthroplasty are influenced differently by patients' body mass index. Knee Surg Sports Traumatol Arthrosc 2018; 26: 3257-64.

34. Lizaur A, Marco L, Cebrian R. Preoperative factors influencing the range of movement after total knee arthroplasty for severe osteoarthritis. J Bone Joint Surg Br 1997; 79: 626-9.

35. Kawamura H, Bourne RB. Factors affecting range of flexion after total knee arthroplasty. J Orthop Sci 2001; 6: 248-52.

36. Wohlrab D, Hube R, Zeh A, Hein W. Clinical and radiological results of high flex total knee arthroplasty: a 5 year follow-up. Arch Orthop Trauma Surg 2009; 129: 21-4.

37. Seon JK, Song EK, Lee JY. Comparison of range of motion of high-flexion prosthesis and mobile-bearing prosthesis in total knee arthroplasty. Orthopedics 2005; 28: s1247-50.

38. Rhee SJ, Hong SM, Suh JT. High-Flexion Total Knee Arthroplasty Using NexGen LPS-Flex System: Minimum 5-year Follow-up Results. Knee Surg Relat Res 2015; 27 : 156-62.

39. Kim Y-H, Sohn K-S, Kim J-S. Range of motion of standard and high-flexion posterior stabilized total knee prostheses. A prospective, randomized study. J Bone Joint Surg. Am 2005; 87: 1470-5.

40. Koh Y-G, Son J, Kwon O-R, Kwon SK, Kang K-T. Effect of Post-Cam Design for Normal Knee Joint Kinematic, Ligament, and Quadriceps Force in Patient-Specific Posterior-Stabilized Total Knee Arthroplasty by Using Finite Element Analysis. BioMed Res Int 2018; 2018: 2438980.

41. Clement ND, Bardgett M, Weir D, Holland J, Gerrand C, Deehan DJ. What is the Minimum Clinically Important Difference for the WOMAC Index After TKA? Clin Orthop Relat Res 2018; 476: 2005-14.

42. Alattas SA, Smith T, Bhatti M, Wilson-Nunn D, Donell S. Greater pre-operative anxiety, pain and poorer function predict a worse outcome of a total knee arthroplasty. Knee Surg Sports Traumatol Arthrosc 2017; 25: 3403-10.

43. Verra WC, van den Boom LGH, Jacobs WCH, Schoones JW, Wymenga AB, Nelissen RGHH. Similar outcome after retention or sacrifice of the posterior cruciate ligament in total knee arthroplasty. Acta Orthop 2015; 86: 195-201.

44. Huang H-T, Su JY, Wang G-J. The early results of highflex total knee arthroplasty: a minimum of 2 years of follow-up. J Arthroplasty 2005; 20: 674-9.

45. Kornuijt A, Kort GJL de, Das D, Lenssen AF, van der Weegen W. Recovery of knee range of motion after total knee arthroplasty in the first postoperative weeks: poor recovery can be detected early. Musculoskelet Surg 2019.

46. Schwarzkopf R, Brodsky M, Garcia GA, Gomoll AH. Surgical and Functional Outcomes in Patients Undergoing Total Knee Replacement With Patient-Specific Implants Compared With "Off-the-Shelf" Implants. Orthop Sports Med 2015; 3: 2325967115590379.

47. Beal MD, Delagrammaticas D, Fitz D. Erratum to: Improving outcomes in total knee arthroplasty-do navigation or customized implants have a role? J Orthop Surg Res 2016; 11: 114 .

48. Parratte S, Pagnano MW, Trousdale RT, Berry DJ. Effect of postoperative mechanical axis alignment on the fifteen-year survival of modern, cemented total knee replacements. J Bone Joint Surg Am 2010; 92: 2143-9.

49. Thienpont E, Cornu O, Bellemans J, Victor J. Current opinions about coronal plane alignment in total knee arthroplasty: A survey article. Acta Orthop Belg 2015; 81: 471-7.

50. Ritter MA, Faris PM, Keating EM, Meding JB. Postoperative alignment of total knee replacement. Its effect on survival. Clin Orthop Relat Res 1994: 153-6.

51. Jones GG, Logishetty K, Clarke S, et al. Do patientspecific instruments (PSI) for UKA allow non-expert surgeons to achieve the same saw cut accuracy as expert surgeons? Arch Orthop Trauma Surg 2018; 138: 1601-8. 